\title{
Determination of Perillic Acid in Bioconversion Supernatants by Gas Chromatography
}

\author{
Eliane G. Carvalho', Antonio C. Siani' ${ }^{2}$, Igor C. Cardoso ${ }^{2}$, Virginia Garcia Correia ${ }^{1}$, \\ Maria A. Ferrara ${ }^{2}$, Elba P. S. Bon², Marcelo R. Romero Tappin ${ }^{*}$ \\ ${ }^{1}$ Medicine and Drug Technology Institute, Oswaldo Cruz Foundation, Rio de Janeiro, Brazil \\ ${ }^{2}$ Chemistry Institute, Federal University of Rio de Janeiro, Rio de Janeiro, Brazil \\ Email: *marcelorrt@fiocruz.br, *siani@far.fiocruz.br
}

How to cite this paper: Carvalho, E.G., Siani, A.C., Cardoso, I.C., Correia, V.G., Ferrara, M.A., Bon, E.P.S. and Tappin, M.R.R. (2017) Determination of Perillic Acid in Bioconversion Supernatants by Gas Chromatography. American Journal of Analytical Chemistry, 8, 636-644. https://doi.org/10.4236/ajac.2017.810046

Received: August 24, 2017

Accepted: October 23, 2017

Published: October 26, 2017

Copyright $\odot 2017$ by authors and Scientific Research Publishing Inc. This work is licensed under the Creative Commons Attribution International License (CC BY 4.0).

http://creativecommons.org/licenses/by/4.0/

\begin{abstract}
Perillic acid can be obtained from microbial oxidation of the exocyclic methyl group of limonene. Due to the pharmacological potential of such a metabolite, the biotransformation processes leading to its synthesis have been approached in recent studies. A robust analytical method is needed to assess the performance of such studies. An analytical method was developed and validated to determine perillic acid in the supernatants of a yeast-induced bioconversion of limonene, involving gas chromatography (GC) and an acid-induced precipitation during the sample preparation. GC analysis was performed using a column with polyethylene glycol as stationary phase (HP-Innowax) which resulted in higher loads and better peak shape. The sample preparation involved the supernatant initial filtration and precipitation with $0.6 \mathrm{M} \mathrm{HCl}$ followed by centrifugation and dissolution in ethyl acetate. GC analysis conditions were oven from $50^{\circ} \mathrm{C}$ to $250^{\circ} \mathrm{C}$ at $20^{\circ} \mathrm{C} \cdot \mathrm{min}^{-1}$, and then held $5 \mathrm{~min}$ (total runtime $15 \mathrm{~min}$ ). Injector was set at $280^{\circ} \mathrm{C}$, and detector at $300^{\circ} \mathrm{C}$. Helium was the carrier gas at $1 \mathrm{ml} \cdot \mathrm{min}^{-1}$. Injections of $1.0 \mu \mathrm{l}$ were at the split ratio $25: 1$. The method was validated: Linearity with $\mathrm{R}^{2}$ of 0.9992 , Accuracy of $98.3 \%$ in the range $190-950 \mu \mathrm{g} \cdot \mathrm{ml}^{-1}$; Limit of detection of $10.4 \mu \mathrm{g} \cdot \mathrm{ml}^{-1}$; Repeatability of $2.1 \%$ RSD. Thus, a complete methodology to determine perillic acid in a bioconversion supernatant was developed and validated. This overall approach may be useful for bioconversions of monoterpenes by other microorganisms that metabolize limonene.
\end{abstract}

\section{Keywords}

Limonene, Perillic Acid, Sample Preparation, Gas Chromatography

\section{Introduction}

The monoterpene (R)-(+)-limonene is the major constituent of citrus peels es- 
sential oil and can be easily extracted from this source [1]. As such, it represents an abundant and relatively inexpensive raw material for producing valuable chemicals. Compounds derived from oxidation at the C-7 position in the limonene moiety are known as the perillic derivatives, e.g. perillyl alcohol, perillyl aldehyde and perillic acid [2] [3]. As the limonene itself, perillyl alcohol and perillic acid are known to have anticancer [2] [4] [5] and anti-inflammatory properties [6]. However, acidic compounds tend to have higher bioavailability [7] and, unlike limonene and perillyl alcohol that are viscous liquids at room temperature, perillic acid is a solid crystal, a property that generally favors studies on drug formulation. Additionally, perillic acid is the main metabolite found in the blood plasma after administering limonene or perillyl alcohol to patients [3]. Reasons such as these have led researchers to assay viable routes to industrial microbial bioconversion of terpenes to perillic acid. Thus, starting from limonene, the perillic acid has been produced by a solvent-resistant Pseudomonas putida strain [8] as well as several yeasts and yeast-like fungi [9] [10]. Monoterpene bio-oxidation by microorganisms usually ends up with the perillic acid dissolved in the bioconversion broth supernatant, which requires a reliable method to quantify such a product in this medium.

In this context, experimental conditions for the limonene bioconversion by the yeast Yarrowia lipolytica have been optimized [11] aiming at further scale up of the process. Therefore, this study aimed at developing a fast and specific methodology to determinate perillic acid amounts in supernatants of limonene bioconversion by Yarrowia lipolytica that included sample preparation by acidic precipitation and gas chromatography (GC) analysis.

\section{Material and Methods}

\subsection{Solvents and Reagents}

$R$-(+)-limonene 97\% was purchased from Sigma (article 183,164). HPLC grade ethyl acetate from Tedia (Fairfield, USA) was used to prepare samples and references standards. Analytical grade $\mathrm{HCl}$ was bought from Vetec (Duque de Caxias, Brazil). (S)-(-)-Perillic acid 95\% from Sigma-Aldrich (St. Louis, USA) was used as reference for perillic acid determination.

\subsection{Apparatus}

Development and most validation analyses of the GC method were carried out in an Agilent Technologies instrument model 7890 equipped with a 7683 injector and autosampler, split/splitless injector, and flame ionization detector (GC-FID) (Santa Clara, USA). Selectivity analysis was carried out in an Agilent Technologies instrument model 6890N coupled with an Agilent Technologies mass spectrometer model 5793N (Santa Clara, USA). A J\&W Scientific HP-Innowax column with a bonded polyethylene glycol phase, $30 \mathrm{~m}$ length, $250 \mu \mathrm{m}$ internal diameter and $0.250 \mu \mathrm{m}$ film thickness, was used in both instruments. A J\&W Scientific DB-5 column with bonded phenyl(5\%)-methyl(95\%)-polysiloxane 
phase, $30 \mathrm{~m}$ length, $250 \mu \mathrm{m}$ internal diameter and $0.250 \mu \mathrm{m}$ film thickness, was used in the first instrument for method development. Sonication was carried out in a Branson sonicator model 5510 (Danbury, USA) and centrifugation in an Eppendorf centrifuge model 5415D (Hamburg, Germany).

\subsection{Bioconversion Supernatant Sample}

The samples analyzed were supernatants obtained by centrifuging limonene bioconversion medium. Bioconversion was carried out by $Y$. lipolytica as described elsewhere [11]. The produced samples were preserved at $-20^{\circ} \mathrm{C}$ until the analysis.

\subsection{Sample Preparation Procedure}

Before analysis, all samples stored as described above were allowed to defrost at room temperature and then homogenized. They were cleared of debris by centrifugation during 15 minutes at 15,000 RCF. An aliquot of $1.0 \mathrm{ml}$ from the supernatant sample was transferred to a $1.7 \mathrm{ml}$ microcentrifuge tube (tube 1). A $0.6 \mathrm{~N} \mathrm{HCl}$ solution was prepared by diluting $2.6 \mathrm{ml}$ of $\mathrm{HCl}$ to $500 \mathrm{ml}$ of deionized water. The acid concentration was standardized by titrating against a sodium hydroxide solution previously standardized with sodium hydrogen phthalate. Perillic acid was precipitated by adding $200 \mu \mathrm{l}$ of $0.6 \mathrm{M} \mathrm{HCl}$ and the resulting suspension was homogenized and centrifuged at 15,000 RCF for 15 minutes. The supernatant was transferred to another $1.7 \mathrm{ml}$ microcentrifuge tube (tube 2) and the precipitation step was performed again with $100 \mu \mathrm{l}$ of $0.6 \mathrm{M} \mathrm{HCl}$. The precipitates were dissolved in ethyl acetate and transferred to a $2.0 \mathrm{ml}$ volumetric flask, which was completed to its capacity with ethyl acetate. The solution was then homogenized and transferred to an appropriate GC analysis vial. Each sample was prepared in triplicate.

\subsection{Perillic Acid Recovery}

Recovery of the sample preparation procedure was assessed by the standard addition method. Thus, three solutions with concentration of 190, 570 and 950 $\mu \mathrm{g} \cdot \mathrm{ml}^{-1}$ of perillic acid standard were prepared in a bioconversion supernatant with low concentration of perillic acid. From each solution, three $1.0 \mathrm{ml}$ aliquots were prepared as described in Section 2.4, alongside three blank samples; the results from the added samples were corrected by the blank ones and then compared to the expected results.

\subsection{Chromatographic Conditions}

All analyses were performed using a temperature program, constant flow, and split injection. The chromatographic conditions were as follows: oven temperature initiated at $50^{\circ} \mathrm{C}$, temperature increased at $20^{\circ} \mathrm{C} \cdot \mathrm{min}^{-1}$ to $250^{\circ} \mathrm{C}$, held for 5 minutes; run time was 15 minutes. The injector temperature was set at $280^{\circ} \mathrm{C}$, and detector temperature at $300^{\circ} \mathrm{C}$. Helium was used as the carrier gas at a flow 
rate of $1 \mathrm{ml} \cdot \mathrm{min}^{-1}$. Injection volume was $1.0 \mu \mathrm{l}$ and the split ratio was set at 25:1.

\subsection{Method Validation}

The following validation parameters were assessed: linearity, precision, accuracy, selectivity, limit of quantitation and limit of detection. Linearity was calculated by analysis of three perillic acid calibration curves, prepared with six different concentrations, ranging from $95 \mu \mathrm{g} \cdot \mathrm{ml}^{-1}$ to $950 \mu \mathrm{g} \cdot \mathrm{ml}^{-1}$. Each concentration was injected four times. Regression analysis was performed for each curve calculating the linear model. Precision was determined at repeatability level calculated from the analysis of real samples in triplicate. Accuracy was calculated by the standard addition as described in Section 2.6. Selectivity was evaluated by peak purity analysis of a real sample in GC coupled to mass spectroscopy (GC-MS). The limit of quantitation was considered as the limits of the calibration range. Limit of detection for perillic acid was calculated from the calibration model using the following expression:

$$
L O D=3.3 \sigma / S
$$

where $\sigma$ stands for the standard deviation of the intercept and $S$ the slope of the calibration curve. Upon the regression model of the calibration curve calculated for validation parameters, the amount of perillic acid in three real samples was then determined.

\section{Results and Discussion}

\subsection{Optimization of Gas-Chromatographic Method}

The large majority of monoterpene analyses by GC is reported upon DB- 5 columns or similar stationary phases from different manufacturers. Likewise, the few studies involving the determination of perillic acid-usually pharmacokinetic experiments-have employed the same type of column to quantify perillic acid [12]. DB-5 stationary phase is composed of a polisiloxane chain with $95 \%$ methyl and 5\% phenyl side chains. It is known that methyl side chains allow better interaction of the stationary phase with non-polar compounds, such as hydrocarbons, by dispersion forces; otherwise, phenyl side chains allow better interaction of the stationary phase with mildly polar compounds by induced dipole forces [13].

By bearing $5 \%$ phenyl side chains, DB- 5 columns are only able to support extremely low mass loads of acid compounds. The capacity of a GC column to admit high mass loads of perillic acid is demonstrated by the maintenance of symmetrical peak shape over a wide concentration interval, which means linear responses and better separations. As can be seen in Figure 1 upon the injection of solutions at several different concentrations, perillic acid forms an asymmetrical peak having a front deformation in DB- 5 column. This bias can usually be overcome by pre-treating the sample with silylation derivatization reagents [14], which means an additional step in the sample preparation. The amount of perillic acid produced from limonene by a strain of Pseudomonas putida has been 


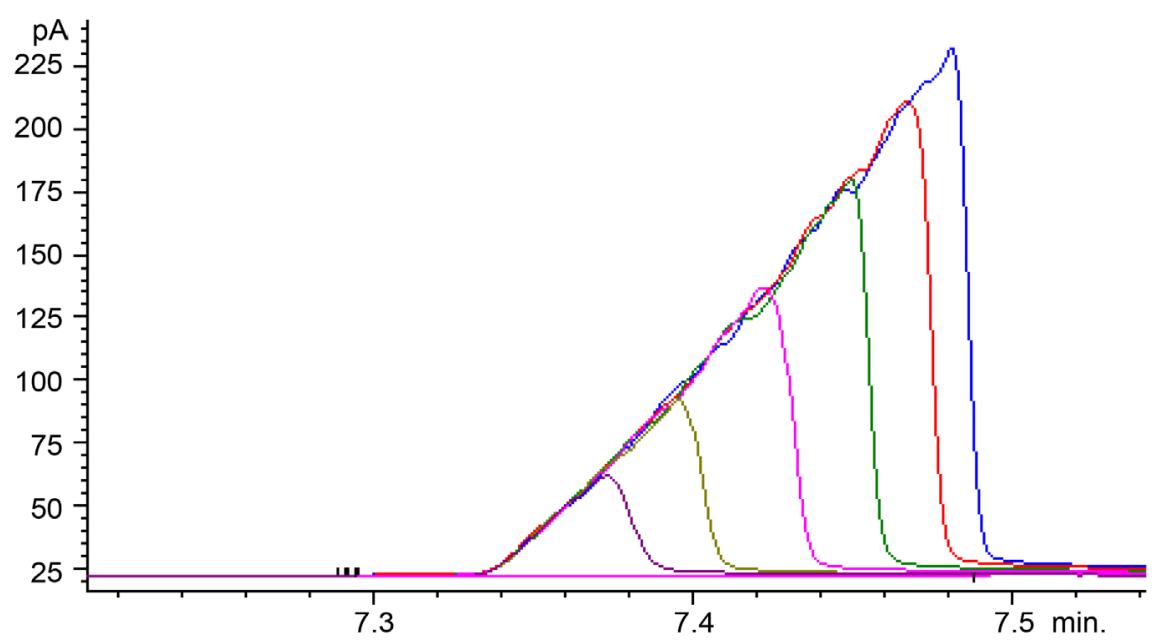

Figure 1. Perillic acid peak shape over several GC-FID injection concentrations in DB-5 column.

monitored by using a CP-58 CB column without any derivatization, but details were not given [8]. The stationary phase of this column is composed by bonded polyethylene glycol modified by nitroterephtalic acid. Polyethylene glycol-based stationary phases are among the most polar stationary phases for GC. The oxygen atoms present in the main chain interacts with acidic functional groups through induced dipole forces, similar to phenyl side chains of DB-5 columns; the difference being a much larger number of interaction points that allow injecting greater mass loads. In order to avoid the additional derivatization step and simplify the sample preparation, a higher-polarity stationary phase was tested. The HP-Innowax column has a polyethylene glycol-based stationary phase and therefore it was tested for perillic acid analysis alongside a DB-5 column. Perillic acid peaks for samples injected in HP-Innowax column retained the symmetric shape for all the tested concentrations, thus indicating its capacity to bear high loads of perillic acid (Figure 2). Therefore, HP-Innowax column was chosen for analyzing perillic acid solutions (Figure 3).

\subsection{Extraction of Perillic Acid and Sample Preparation}

The main difficulty of determining perillic acid in bioconversion supernatant is the aqueous characteristic of this matrix. The challenge is to extract perillic acid from this medium since water is not recommended as solvent in GC analysis. The first option devised was a liquid/liquid extraction by using ethyl acetate. Although initial results indicated that the extraction was feasible, at least $5 \mathrm{ml}$ of supernatant were necessary to achieve proper phase separation; furthermore a series of extractions were necessary to exhaust the perillic acid content from the aqueous phase (data not shown). Since perillic acid was the expected organic compound present in the bioconversion supernatant, treatment with an acid solution, of e.g. $\mathrm{HCl}$, could cause it to precipitate and eventually result in much purer samples for analysis. In the first step, the most suitable $\mathrm{HCl}$ concentration 


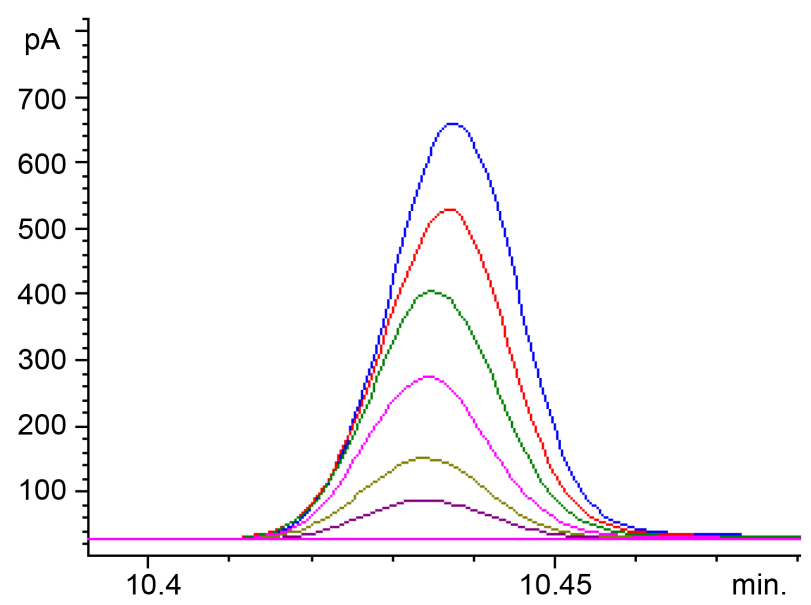

Figure 2. Perillic acid peak shape over several GC-FID injection concentrations in HP-Innowax column.

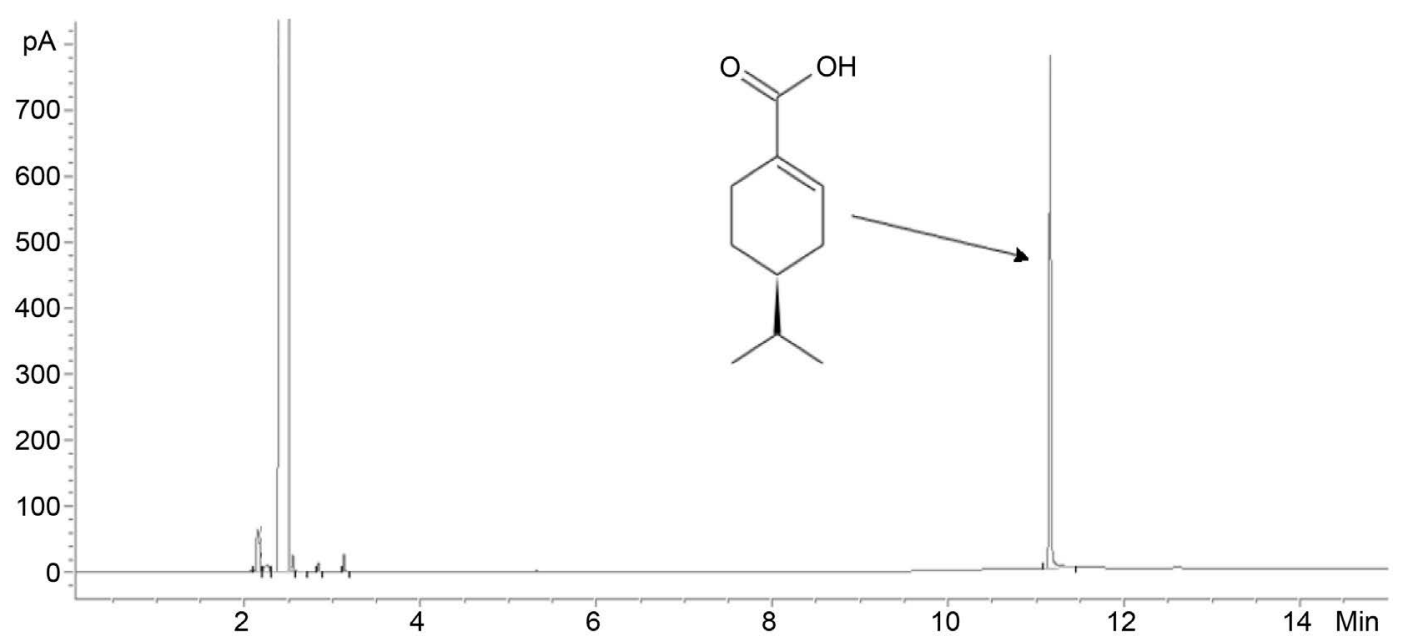

Figure 3. GC-FID chromatogram showing perillic acid $(11.151 \mathrm{~min})$ after applying the sample preparation procedure to a real sample. The remaining peaks correspond to the solvent.

for perillic acid precipitation was determined to be $0.6 \mathrm{M}$. The procedure included acid precipitation, centrifugation and dissolution of the precipitate in ethyl acetate, as described in Section 2.4, to provide suitable samples for GC analysis. To evaluate this procedure, perillic acid recovery from bioconversion supernatant was assessed by adding known concentrations of perillic acid standard to a supernatant sample with low concentration of the analyte. Acidic precipitation is often dependent on the concentration of the targeted compound, thus three concentrations were prepared and tested to ensure recovery: 190, 570 and $950 \mu \mathrm{g} \cdot \mathrm{ml}^{-1}$. The perillic acid recovery was adequate, with the worst value (95.2\%) being observed at $950 \mu \mathrm{g} \cdot \mathrm{ml}^{-1}$. Analysis of variance was applied to compare the resulting concentrations in the three cases (Figure 4). Although there was a decreasing trend among the results toward higher concentrations, the ANOVA test failed to detect significant differences between levels, therefore the recovery was considered the mean value of all the results, which was $98.3 \%$. 


\subsection{Method Validation}

Linearity was proven by three separate curves; the least determination coefficient was found to be 0.9992 . In the calibration curve (data not shown), the regression analysis also pointed out the intercept to be significantly different from zero, indicating the need to prepare a full calibration curve for each analysis. Accuracy was determined by recovery tests during the development of the sample preparation procedure as discussed above, with the ultimate result attaining $98.3 \%$. The relative standard deviation of real samples were calculated and presented in Table 1.

Repeatability was considered $2.11 \%$ as it was the larger RSD value. The selectivity was assessed by peak purity analysis of a real sample in MSD chemstation enhanced data analysis (version D.02.00.275 Agilent Technologies) and the results indicated no compounds co-eluting with perillic acid. Limit of detection was calculated as $10.4 \mu \mathrm{g} \cdot \mathrm{ml}^{-1}$ from the mathematical model of the calibration curves, which is far below the lowest concentration of the curve. The overall developed methodology was successfully applied to several bioconversion supernatant samples, showing to be suitable to quantify perillic acid produced from limonene or orange oil employing Y. lipolytica [11], as shown in Table 1.

\section{Conclusions}

A complete methodology for determination of perillic acid in bioconversion supernatants was developed and successfully validated. It included a high-resolution gas chromatography method and a sample preparation procedure. This method

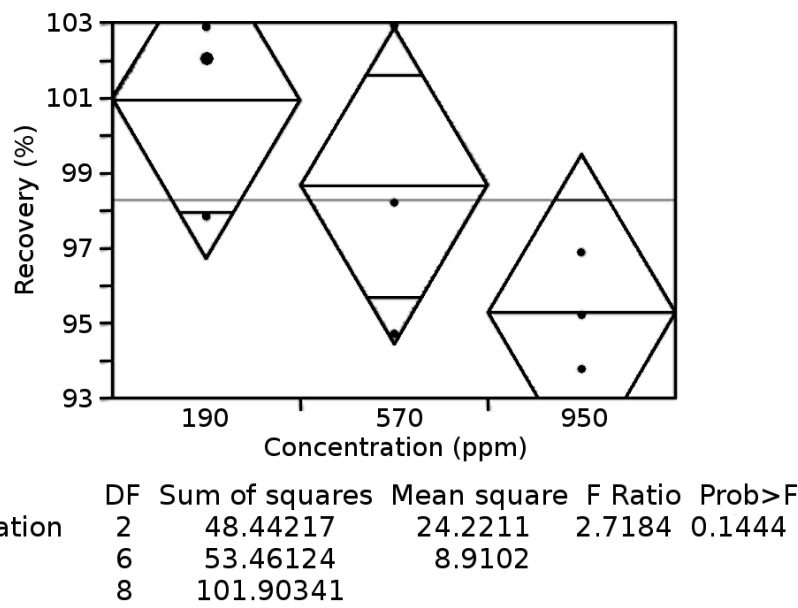

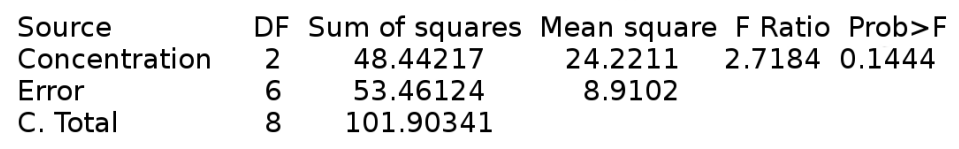

Figure 4. Analysis of variance for perillic acid recovery.

Table 1. Perillic Acid (PA) determination on real samples.

\begin{tabular}{ccc}
\hline Starting material & Measured PA & RSD (\%) \\
\hline Orange oil (89.1\% limonene) & $866 \mathrm{ppm}$ & 1.16 \\
Commercial limonene (97\%) & $862 \mathrm{ppm}$ & 0.52 \\
Commercial limonene (97\%) & $879 \mathrm{ppm}$ & 2.10 \\
\hline
\end{tabular}


can be applied to support the development and quality control of perillic acid production processes by limonene bioconversion.

\section{Acknowledgements}

The authors thank to FAPERJ-RJ/Brazil for financial support (proc. E-26/110.856/2013-APQ1).

\section{References}

[1] Duetz, W.A., Bouwmeester, H., Van Beilen, J.B. and Witholt, B. (2003) Biotransformation of Limonene by Bacteria, Fungi, Yeasts, and Plants. Applied Microbiology and Biotechnology, 61, 269-277. https://doi.org/10.1007/s00253-003-1221-y

[2] Bicas, J.L., Fontanille, P., Pastore, G.M. and Larroche, C. (2008) Characterization of Monoterpene Biotransformation in Two Pseudomonas. Journal of Applied Microbiology, 105, 1991-2001. https://doi.org/10.1111/j.1365-2672.2008.03923.x

[3] Chow, H-H.S., Salazar, D. and Hakim, I.A. (2002) Pharmacokinetics of Perillic Acid in Humans after a Single Dose Administration of a Citrus Preparation Rich in D-Limonene Content. Cancer Epidemiology, Biomarkers \& Prevention, 11, 1472-1476.

[4] Bardon, S., Picard, K. and Martel, P. (1998) Monoterpenes Inhibit Cell Growth, Cell Cycle Progression and Cyclin D1 Gene Expression in Human Breast Cancer Cell Lines. Cancer Letters, 32, 1-7. https://doi.org/10.1080/01635589809514708

[5] Yeruva, L., Pierre, K.J., Elegbede, A., Wang, R.C. and Carper, S.W. (2007) Perillyl Alcohol and Perillic Acid Induced Cell Cycle and Apoptosis in Non-Small Cell Lung Cancer. Cancer Letters, 257, 216-226. https://doi.org/10.1016/j.canlet.2007.07.020

[6] Schulz, S., Reinhold, D., Schmidt, H., Ansorge, S. and Höllt, V. (1997) Perillic Acid Inhibits Ras/MAP Kinase-Driven IL-2 Production in Human T Lymphocytes. Biochemistry and Biophysical Research Communication, 241, 720-725. https://doi.org/10.1006/bbrc.1997.7884

[7] Andrews, C.W., Bennett, L. and Yu, L.X. (2000) Predicting Human Oral Bioavailability of a Compound: Development of a Novel Quantitative Structure-Bioavailability Relationship. Pharmaceutical Research, 17, 639-644. https://doi.org/10.1023/A:1007556711109

[8] Speelmans, G., Bijlsma, A. and Eggink, G. (1998) Limonene Bioconversion to High Concentrations of a Single and Stable Product, Perillic Acid, by a Solvent-Resistant Pseudomonas putida Strain. Applied Microbiology and Biotechnology, 50, 538-544. https://doi.org/10.1007/s002530051331

[9] Van Rensburg, E., Moleleki, N., Van der Walt, J.P., Botes, P.J. and Van Dyk, M.S. (1997) Biotransformation of (+)-Limonene and (-)-Piperitone by Yeasts and Yeast-Like Fungi. Biotechnology Letters, 19, 779-782. https://doi.org/10.1023/A:1018344411069

[10] Ferrara, M.A., Almeida, D.S., Siani, A.C., Lucchetti, L., Lacerda, P.S.B., Freitas, A., Tappin, M.R.R., et al. (2013) Bioconversion of R-(+)-Limonene to Perillic Acid by the Yeast Yarrowia lipolytica. Brazilian Journal of Microbiology, 44, 1075-1080. https://doi.org/10.1590/S1517-83822014005000008

[11] Tappin, M.R.R., Knopp, F.M., Cardoso, I.C., Santos, R.T., Drummond, B.S., Siani, A.C., Bon, E.P.S. and Ferrara, M.A. (2017) Synthesis of the Prospective Anticancer Molecule Perillic Acid from Orange Essential Oil by the Yeast Yarrowia lipolytica. Green and Sustainable Chemistry, 7, 172-184. 
https://doi.org/10.4236/gsc.2017.72013

[12] Crowell, P.L., Elson, C.E., Bailey, H.H., Elegbede, A., Haag, J.D. and Gould, M.N. (1994) Human Metabolism of the Experimental Cancer Therapeutic Agent D-Limonene. Cancer Chemotherapy and Pharmacology, 35, 31-37. https://doi.org/10.1007/BF00686281

[13] Matei, V., Comănescu, L. and Borcea, A.-F. (2012) Stationary Phases. In: Mohd, M.A., Ed., Advanced Gas Chromatography-Progress in Agricultural, Biomedical and Industrial Applications, InTech, Rijeka, 27-50. https://doi.org/10.5772/34152

[14] Zhang, Z., Chen, H., Chan, K.K., Budd, T. and Ganapathi, R. (1999) Gas Chromatographic-Mass Spectrometric Analysis of Perillyl Alcohol and Metabolites in Plasma. Journal of Chromatography B: Biomedical Science Applied, 728, 85-95. https://doi.org/10.1016/S0378-4347(99)00065-1 